\title{
Study on various excitation voltage effects to the transient responses of a single long horizontal ground conductor
}

\begin{abstract}
This study is carried out to determine a suitable excitation source that can be used to model the lightning return stroke on a single long horizontal ground conductor. It is used to determine the fastest results obtained for the transient response of a single long horizontal ground conductor and for computation efficiency purposes. In this study, three various excitation sources are used and been compared to each other; that are derivative Gaussian, double exponential and one-cycle sinusoidal functions. The transient node voltages and transient node currents due to the lightning strike are obtained using TLM method. It is found that the derivative Gaussian excitation source demonstrates the fastest results compared to the other two. However, if a study is for observing the behaviour of lightning return stroke, a double exponential excitation source is more suitable.
\end{abstract}

Keyword: Derivative Gaussian; Double exponential; Impulse excitation voltage; Single long horizontal ground conductor; Sinusoidal; TLM; Transient responses 